\title{
VASCULAR PATTERN CHANGES IN ZHE HEART DUE TO TRAINING
}

Yoshio OGAWA, M.D.

Institute of Sports Medicine, Yokohama City University

It is generally said that the angioarchitectures in the cardiac wall are very complicated and are not clear in detail. Because of this, it is important to study the distribution of the minute blood vessels in the cardiac wall for understanding the coronary blood flow in the heart.

After endurance exercises in the developing stage, the hypertrophy of the cardiac wall is often observed as the so-called sports heart. The hypertrophy is often found in the muscle layers of the inner side of the left ventricular wall. As a result, the muscle layers of the inner side of the left ventricular wall will need an abundant blood supply and also the changes in the micro-vascular patterns will be found.

The purpose of this paper is to report the changes of the vascular patterns in the ventricular wall of the albino rat after endurance exercise.

\section{Methods}

The experiments were conducted to observe the micro-vascular pattern changes of the albino rat's heart after being given swimming exercises for 60 minutes a day for 60 days starting 150 days from birth. Three hundred male rats were equally divided into two groups, an exercised group and a non-exercised group. After birth all the rats lived under the same conditions. The growth curve of the rats being used in the experiments is shown in Fig. 1. During the swimming exercise period the body weights of the exercised group were comparatively light compared to the non-exercised group, but the heart weights were always heavier. The results are shown in Fig. 2.

At the end of the swimming exercises, heart-lung preparations were made from both groups. At first, Ringer's solution was perfused into the blood stream, and then Indian ink Ringer's solution was continuously injected. Morphological investigations were made with Indian ink injected hearts. The minute blood vessels of the hearts were filled with Indian ink Ringer's solution.

\section{Results}

The ventricular wall was divided into four parts from the base to the apex, and each part was examined for vascular pattern changes. The main changes of the micro-vascular arrangements in the ventricular wall were observed in the second part. The muscle archi- 


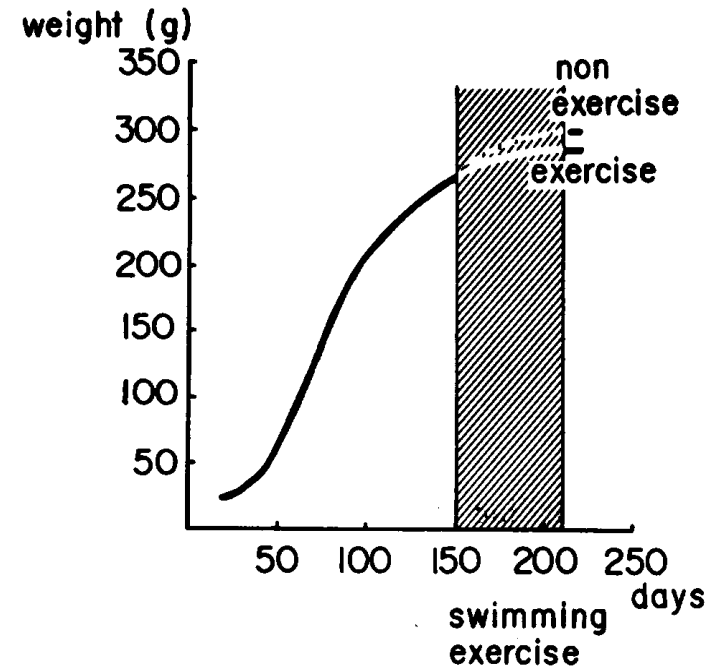

Fig. 1. Graphic growth record of the rat.

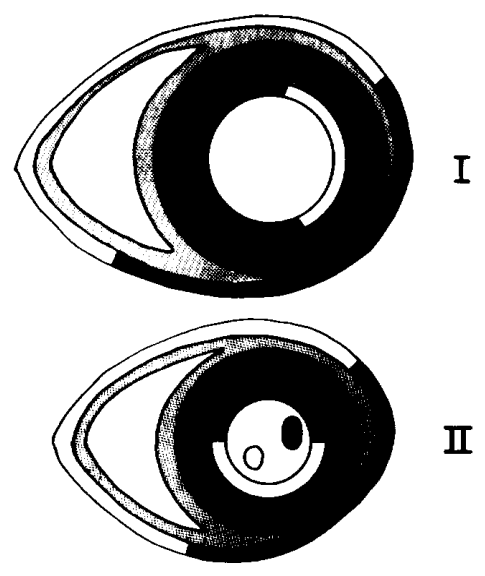

III

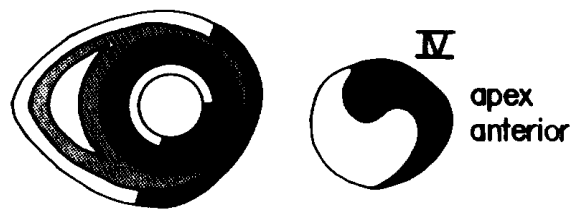

Superficial bulbospiral

Superficial sinospiral

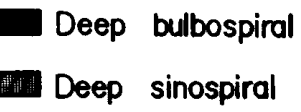

Fig. 3. Muscle architecture of the ventricle.

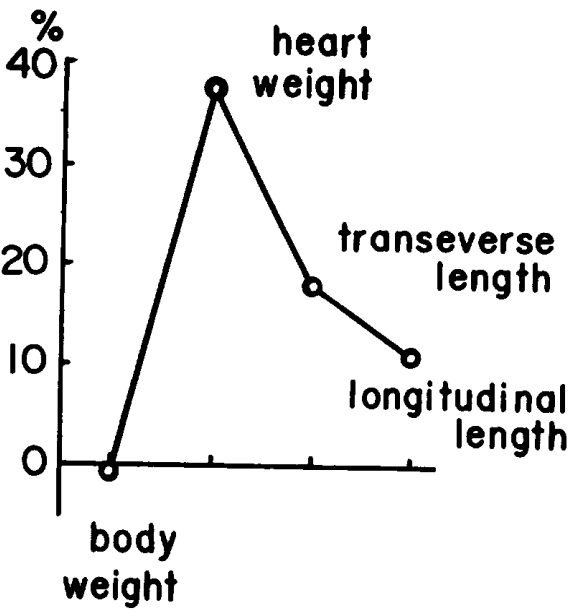

Fig. 2. Increment of the body weight, the heart weight, and the size of the heart in percent.

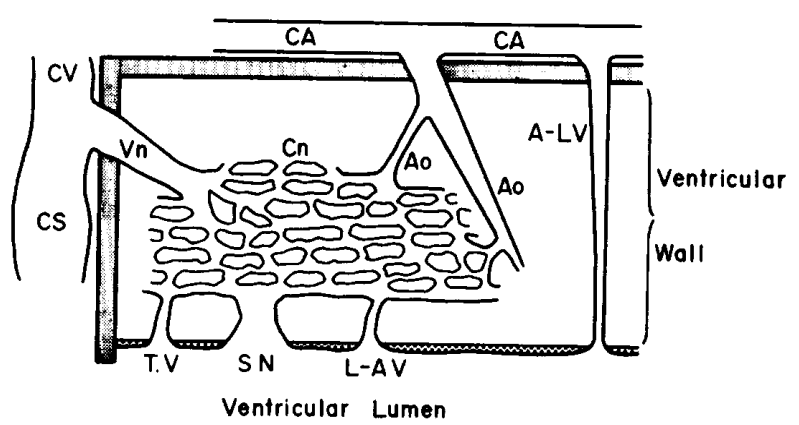

Fig. 4. Vascular arrangements of the ventricular wall.

CA : coronary artery

$\mathrm{CV}$ : coronary vein

CS : coronary sinus

A-LV : arterio luminal vessel

T.V : Thebesian vessel (vein)

$\mathrm{SM}$ : sinusoidal luminal vessel

L-AV : lumina arterial vessel

Ao : arteriole

$\left.\begin{array}{l}\text { Cn }: \text { net-capillary } \\ \text { Vn : venule }\end{array}\right\}$ capillary bed

tecture $^{(7)(10)(12)}$ in the same segments is shown in Fig. 3.

Fig. 4 shows a schema of the blood vascular arrangements of the ventricular wall. Five characteristic features are shown in this schematic drawing.

I. Capillary bed,

II. Thebesian vessels,

III. Sinusoidal-luminal vessels,

IV. Communications between the left ventricular lumen and the layer of the left 


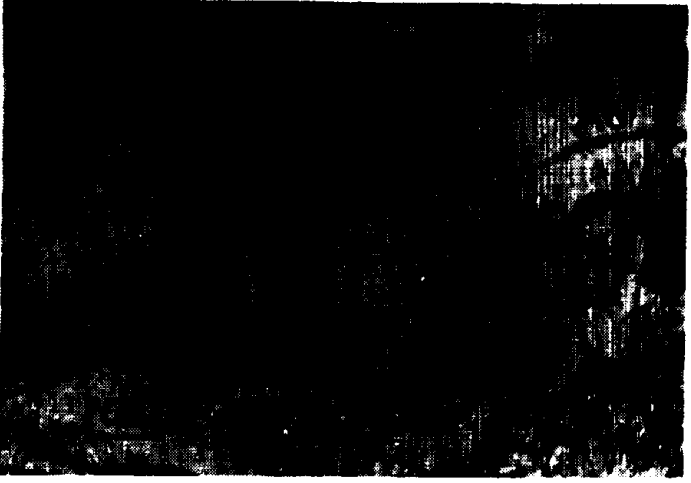

Fig. 5. Distribution of the minute blood vessel in the left ventricular wall.

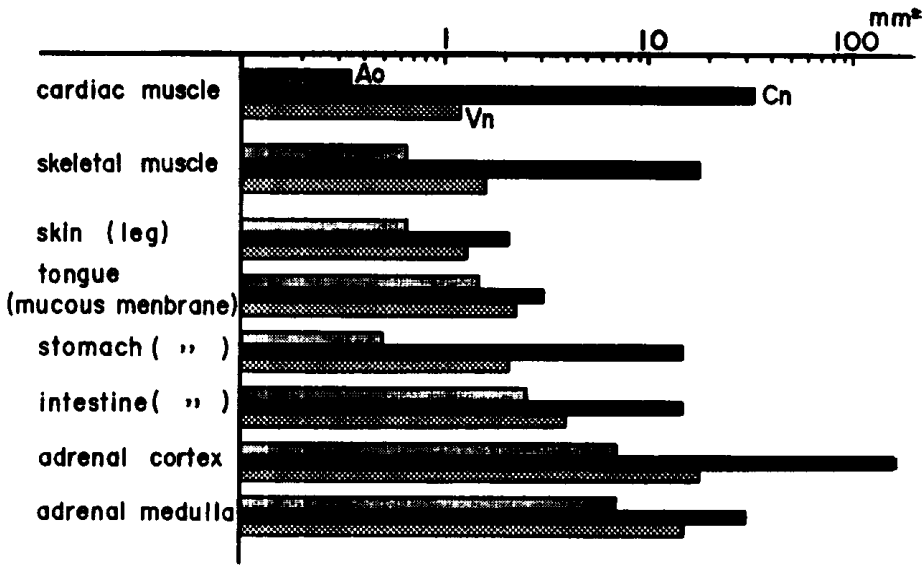

Fig. 6. Surface area of minute blood vessels per one cubic meter in volume of the organs.

ventricular wall (newly named the lumina arterial vessels),

V. Arterio-luminal vessels (Penetrate into the ventricular wall and connect with the ventricular lumen. However, no arterio-luminal vessels were found in the ventricular walls of the rats).

The above four items were investigated in this report. Fig. 5 shows a ventricular wall in the injected specimen of the non-exercised group. There are a lot of capillary networks in the muscle layers of the left ventricular wall, but the numbers of the afferent arteriole are few, and the efferent venules, dilating and running tortously, are numerous compared to the afferent arteriole.

The histogram (Fig. 6) shows the differences of the vascularity of the various kind of organs. The vascularity is indicated by the surface areas of the minute blood vessels. In the left ventricle, the surface areas of the net-capillary are very wide, but on the contrary, those of afferent arteriole are not so wide.

The micro-vascular arrangements of the right ventricular wall in the injected specimen of the non-exercised group are shown in Fig. 7. The peripheral branch of the coronary small artery ramifies and distributes from the surface to the deep layers. The right side of Fig. 7 and Fig. 8 is the right ventricular lumen and in the middle part of Fig. 8, the sinusoidal luminal vessels can be seen. The capillary networks are dense, particularly in the inner side, the venules distribute regularly and have large caliber.

Fig. 9 shows the minute blood vessel distribution in the septum wall of the non-exercised group, the vacant space is the right ventricular lumen. The vein running into the right ventricular lumen can be observed. This is usually called the Thebesian vessel. These vessels are mainly found in the septum wall. The capillary networks in the septum wall are comparatively dense and there are no differences in the vascular pattern between the right and the left side of the septum wall.

The dorsal wall of the ventricular wall of the non-exercised group is shown in Fig. 10. The right side is the right ventricle, the left is the left ventricle and middle is the dorsal 


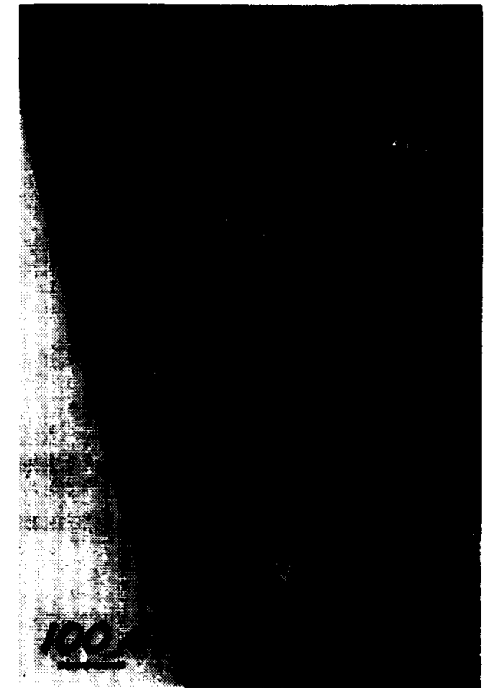

Fig. 7. Distribution of the minute blood vessel in the right ventricular wall.

$\mathrm{RL}$ : right ventricular lumen

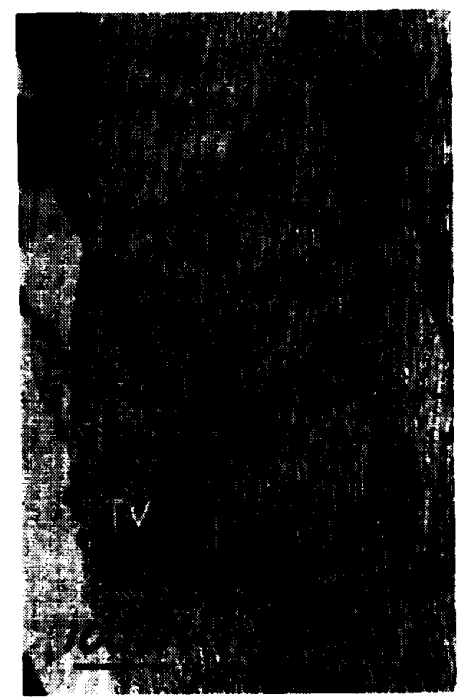

Fig. 9. Distribution of the minute blood vessel in the septum wall.

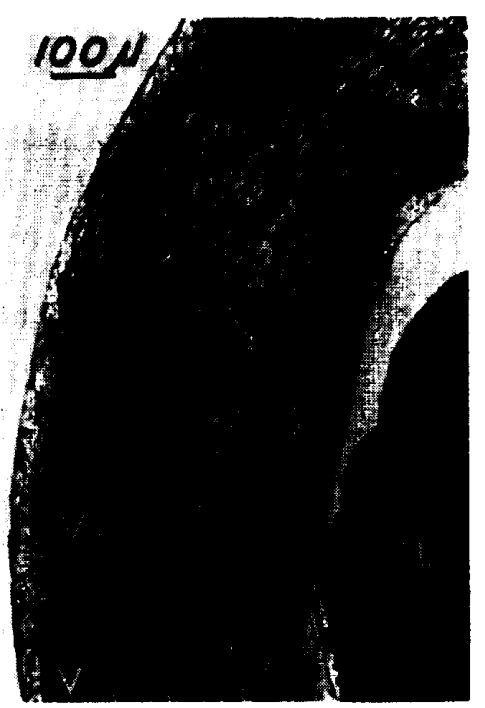

Fig. 8. Distribution of the minute blood vessel in the right ventricular wall.

$\mathrm{RL}$ : right ventricular lumen

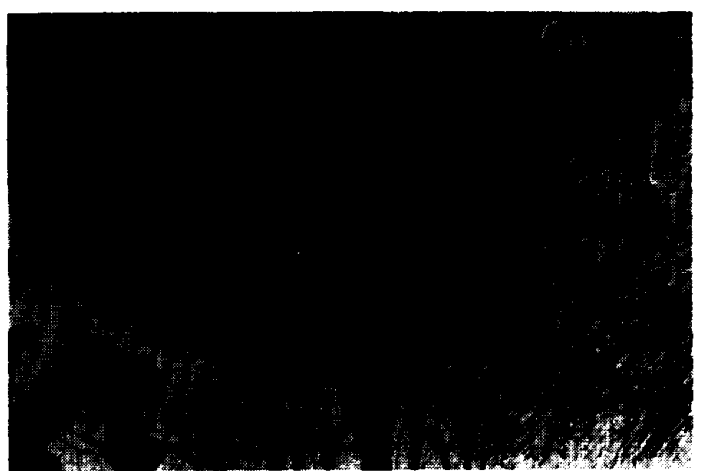

Fig. 10. Distribution of the minute blood vessel in the posterior cardiac wall.

$\mathrm{RL}$ : right ventricular lumen

side of the septum wall. In the right ventriclar wall of the septum, the large caliber veins have joined into the right ventricular lumen. These are called sinusoidal luminal vessels.

The distribution of the minute blood vessels in the left ventricular wall of the non-exercised group is shown in Fig. 11. The vascular arrangements in the left ventricular wall are apparently different from the surface and the middle or the deep layers. The vascularity of the surface layer is plentiful, but in the middle or deep layers it is sparse.

The relative vascular density of the middle part of the ventricular wall measured by the densitometer are shown in Fig. 12. In this diagram, the numbers of the left side show the 


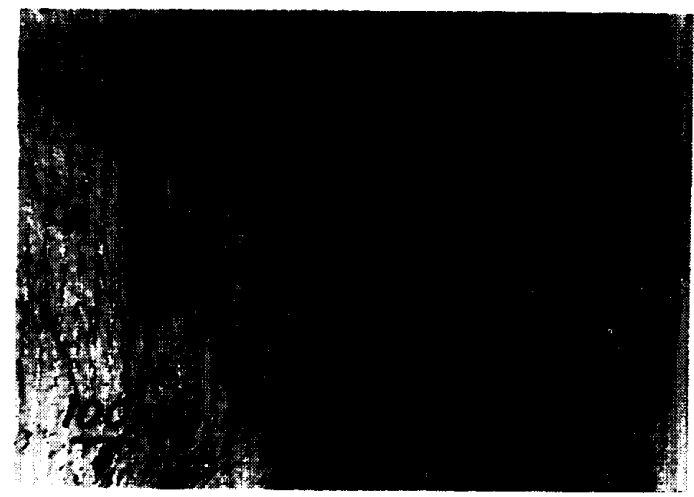

Fig. 11. Distribution of the minute blood vessel in the left ventricular wall.
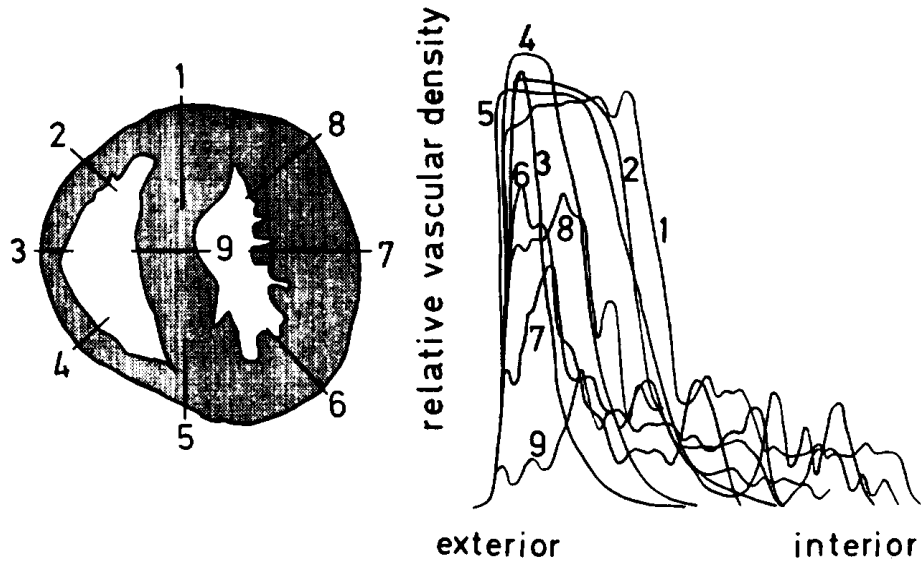

Fig. 12. Relative vascular density curves in the ventricular wall.

parts of the measurement, and the ripples of the right side indicate relative vascular density in each part. Part number 6,7 and 8 of the wall are thick, but the vascular densities in the inner side are sparse compared to that of the outer side.

In the left ventricular wall of the non-exercised group, the vascular arrangements of the surface layer are generally abundant, but the middle or deep layer are sparce.

After endurance exercises, the muscle bundles of the middle or deep layer develop, and the vascularizations of the exercised group apparently different compared to those of the non-exercised group (Fig. 13). Particularly, the vascular communications between the left ventricular lumen and the middle or deep layer of the left ventricular wall clearly increase (Fig. 14).

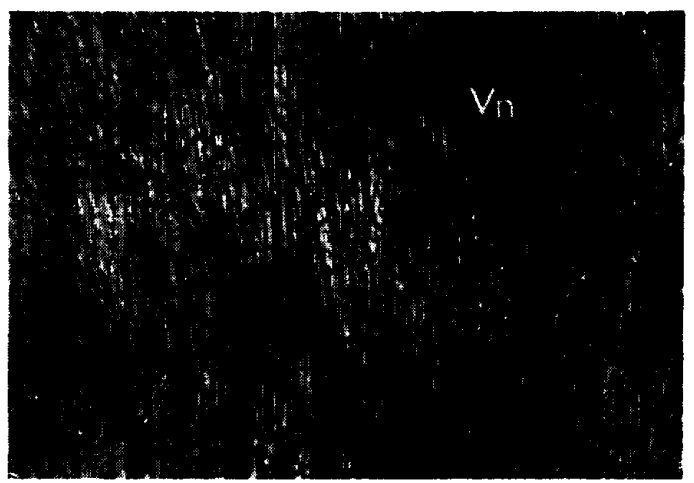

Fig. 13. Distribution of the minute blood vessel in the left ventricular wall. (exercised group)

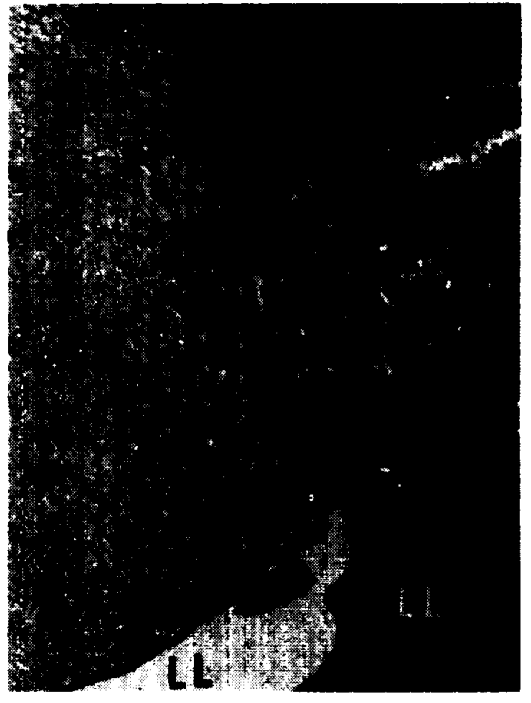

Fig. 14. Distribution of the minute blood vessel in the deep layer of the left ventricle. (exercised group)

LL: left ventricular iumen 


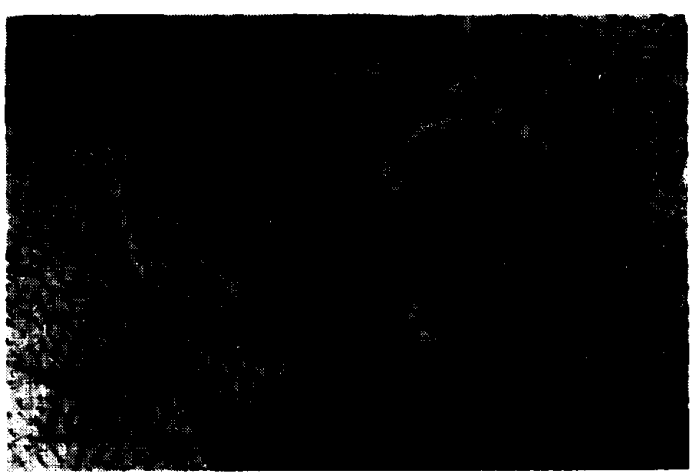

Fig. 15. Vascular communication between the left ventricular lumen and the left ventricular wall. (exercised group)

LL: left ventricular lumen

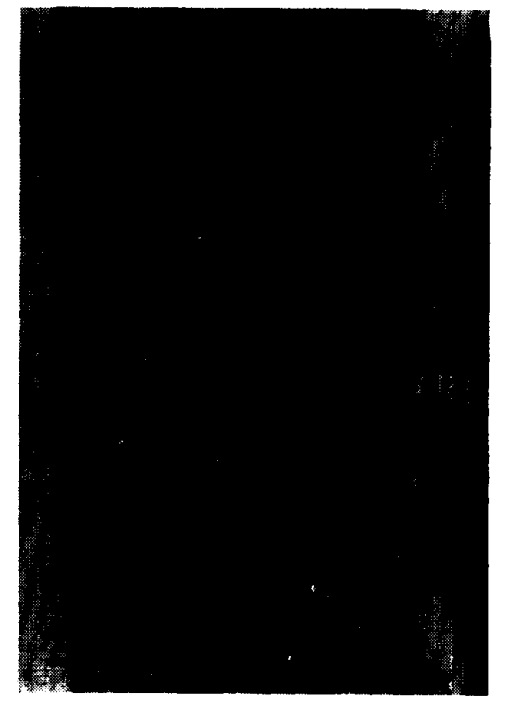

Fig. 16. Distribution of the minute blood vessel in the right ventricular wall. (exercised group)

$R L$ : right ventricular lumen

These vascular communiations should be thought to name newly lumina arterial vessels. When the specimens are stained by the Hematoxylin and Eosin, the lumina arterial vessels, penetrating into the muscle layers and anastomosing the capillary bed, are observed in Fig. 15.

In the right ventricular wall in Fig. 16, the capillaries and the venules generally dilate. The capillary networks are dense. The sinusoidal luminal vessels are developed in the dorsal wall of the right ventricle.

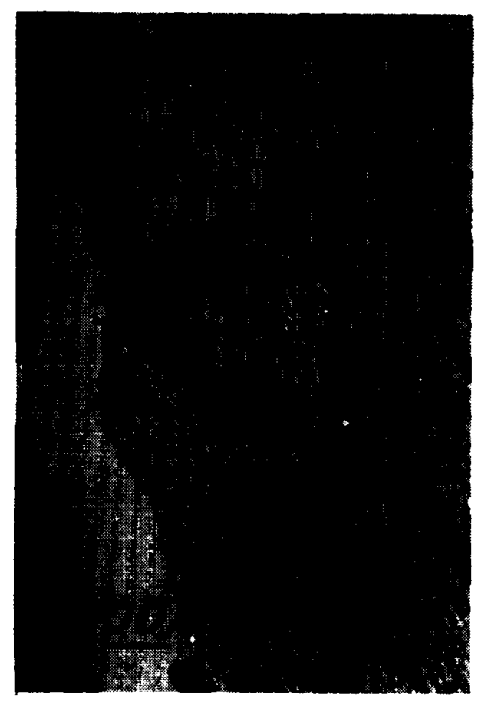

Fig. 17. Distribution of the minute blood vessel in the septum wall. (exercised group) $\mathrm{RL}$ : right vetricular lumen 
In the septum wall in Fig. 17, a lot of the Thebesian vessels, having large caliber and running into the right ventricular lumen, are usually observed.

\section{Discussion}

There are lots of reports of the specific(4)(3)(6)(8)(11) vessels in the cardiac wall, particulary, the existence of the Thebesian vessels and the sinusoidal luminal vessels are well known, but the physilogical functions ${ }^{13)}$ of these vessels are uncertain.

In general, it is said that coronary circulation is similar to that of another vascular beds. It differs, however, in that arteries, capillaries and veins are connected with both ventricular lumens.

According to the report by Gregg(1),2),3) almost all coronary artery inflow can be collected in the superficial veins of the left and right heart, leaving only a small volume to drain into the ventricular lumen in dogs.

In this work, the exsistence of the specific vessels of the cardiac wall were ascertained. Particularly, the finding of the communications between the left ventricular wall after 60-day period of the endurance exercise is important to realize the blood flow in the deep layer of the left ventricular wall.

Fig. 18 is a schematic drawing of the blood flow in the ventricular wall of the arbino rat. Arrow marks in the drawing show the direction of the blood flow in the ventricular wall. Ordinarily, the blood flows along following course; from the branches of the coronary artery to the capillary beds in the ventricular wall and finally into the branches of the coronary vein.

But in the deep layer of the ventricular wall the capillary beds of ten communicate with the right ventricular lumen through the sinusoidal luminal vessels, and the capillary beds in the septum wall communicate with the right ventricular lumen through the Thebesian vessels.

In addition to the micro-vascular communications between the left ventricular wall and

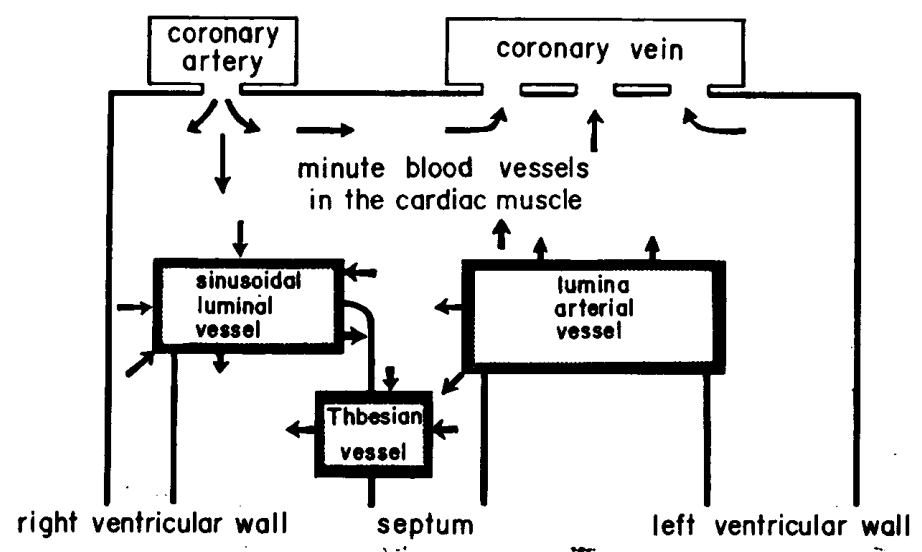

Fig. 18. Vascular arrangements in the ventricular wall, 
the lumen, which I would like to name lumina arterial vessels, are shown after 60-day period of endurance swimming exercises.

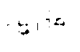

\section{Summary}

An experiment was conducted to observe the blood vascular pattern changes of the ventricular walls in the developing stage of the albino rat's heart, which was given swimming exercises for about 60 minutes a day for a period of 60 days, starting 150 days from birth. The results are obtained as follows.

The venules and the small veins in the surface and middle layers of the ventricular walls of the exercised group were remarkably well developed as compared with the non-exercised group. A great number of venous-sinuses, which are sometimes refered to as sinusoidal luminal vessels were found in the wall of the septum, both in the right and left ventricles. The venous-sinuses of the dorsal wall near the septum were often anastomosed into the lumen of the right ventricle. Some of the venules and the small veins of the septum which are called Thebesian vessels were found to be joined to the lumen of the right ventricle.

A few peripheral branches of the coronary artery were found in the dep layer of the left ventricular wall, however, a lot of vascular connections that I have called lumina arterial vessels were observed between the left ventricular lumen and the deep layer of the wall of the left ventricle. The most interesting result was thought to be the fact that the vascular connections between the left ventricular lumen and the deep layer in the wall of the left ventricle clearly found after 60-day period of the swimming exercises.

\section{References}

1) Gregg, D. E. : The coronary circulation in health and disease. Philadelphia, 1950.

2) Gress, D. E. : Aortic and coronary flow., Visceral circulation, 139-155. J \& A Churchill Ltd., London, 1952.

3) Gregg, D. E. and Fisher, L. : Blood supply to the heart. Handbook of Physiology, Section 2, Circulation, Vol. II. 1517-1584, 1963.

4) lkeda, M. and Murata, K. : Physiology and Pathology of Coronary Circulation (Peripheral Circulation and It's Patho-physiology), 409, Tokyo University Press, Tokyo, 1967. (in Japanese)

5) Ito, Y.: Volume of coronary flow, Respiration and Circulation, 18:53, 1970. (in Japanese)

6) Lictata, R. : Coronary Circulation, Blood Vessels and Lymphatics, 258, Academic Press, New York and London, 1962.

7) Mall, F. P.: On the muscular architecture of the ventricles of the human heart., Amer. J. Anat., 11:211, 1911.

8) Nakamura, T., Suzuki, N. and Hayashi, M. : Physiology of coronary circulation., Saishin Igaku, 18-5, 1963. (in Japanese)

9) Ogawa, Y., Yusa, S., Takahashi, M. and Yamaguchi, H. : Studies of the minute blood vessels (III)., Destribution of minute blood vessels of skeletal muscle., J. Yokohama City Univ., Seri. C-44, 145, 1963. (in Japanese) 
10) Otsu, S. : Coronary Insufficiency. Recent Advance of Basic Medicine, 471, IshiyakuShuppan, Tokyo, 1956. (in Japanese)

11) Plotz, M. : Coronary Heart Disease. New York, 1957.

12) Robb, J. S. and Robb, R. C. : The normal heart., Amer. Heart J. 23 : 455, 1942.

13) Rushmer, R. F., Crystal, D. K. and Wagner, C. : The functional anatomy of ventricular contraction., Circulat. Res., 1 : 162, 1953.

14) Yusa, S. : Study on the coronary vascular system : Distribution of the minute blood vessels in the heart., J. Yokohama City Univ., Seri. C-35, 120, 1, 1966. (in Japanese) 